\title{
A Modular Labeling Strategy for In Vivo PET and Near-Infrared Fluorescence Imaging of Nanoparticle Tumor Targeting
}

\author{
Carlos Pérez-Medina ${ }^{1-3}$, Dalya Abdel-Atti ${ }^{4}$, Yachao Zhang ${ }^{4}$, Valerie A. Longo ${ }^{4}$, Chrisopher P. Irwin $^{4}$, Tina Binderup ${ }^{3,5}$, \\ Jesús Ruiz-Cabello ${ }^{1,2}$, Zahi A. Fayad ${ }^{3}$, Jason S. Lewis ${ }^{4,6,7}$, Willem J.M. Mulder ${ }^{3}$, and Thomas Reiner ${ }^{4,7}$ \\ ${ }^{I}$ Centro de Investigación en Red de Enfermedades Respiratorias, Madrid, Spain; ${ }^{2}$ Centro Nacional de Investigaciones Cardiovasculares, \\ CNIC, Madrid, Spain; ${ }^{3}$ Translational and Molecular Imaging Institute, Icahn School of Medicine at Mount Sinai, New York, New York; \\ ${ }^{4}$ Radiochemistry and Imaging Sciences Service, Department of Radiology, Memorial Sloan-Kettering Cancer Center, New York, \\ New York; ${ }^{5}$ Cluster for Molecular Imaging, Faculty of Health Sciences and Department of Clinical Physiology, Nuclear Medicine and \\ PET, Copenhagen, Denmark; ${ }^{6}$ Molecular Pharmacology and Chemistry Program, Memorial Sloan-Kettering Cancer Center, New York, \\ New York; and ${ }^{7}$ Center for Molecular Imaging and Nanotechnology, Memorial Sloan-Kettering Cancer Center, New York, New York
}

Advances in preclinical molecular imaging have generated new opportunities to noninvasively visualize the biodistribution and tumor targeting of nanoparticle therapeutics. Capitalizing on recent achievements in this area, we sought to develop an ${ }^{89} \mathrm{Zr}$-based labeling strategy for liposomal nanoparticles that accumulate in tumors via passive targeting mechanisms. Methods: ${ }^{89} \mathrm{Zr}$-labeled liposomes were prepared using 2 different approaches: click labeling and surface chelation. Pharmacokinetic and biodistribution studies, as well as $\mathrm{PET} / \mathrm{CT}$ imaging of the radiolabeled nanoparticles, were performed on a mouse model of breast cancer. In addition, a dual PET/optical probe was prepared by incorporation of a near-infrared fluorophore and tested in vivo by PET and near-infrared fluorescence imaging. Results: The surface chelation approach proved to be superior in terms of radiochemical yield and stability, as well as in vivo performance. Accumulation of these liposomes in tumor peaked at $24 \mathrm{~h}$ after injection and was measured to be $13.7 \pm 1.8$ percentage injected dose per gram. The in vivo performance of this probe was not essentially perturbed by the incorporation of a near-infrared fluorophore. Conclusion: We have developed a highly modular and efficient strategy for the labeling of liposomal nanoparticles with ${ }^{89} \mathrm{Zr}$. In xenograft and orthotopic mouse models of breast cancer, we demonstrated that the biodistribution of these nanoparticles can be visualized by PET imaging. In combination with a near-infrared dye, these liposomal nanoparticles can serve as bimodal PET/optical imaging agents. The liposomes target malignant growth, and their bimodal features may be useful for simultaneous PET and intraoperative imaging.

Key Words: liposomes; ${ }^{89} \mathrm{Zr}$; PET; near-infrared imaging; breast cancer

J Nucl Med 2014; 55:1706-1711

DOI: 10.2967/jnumed.114.141861

Received Apr. 20, 2014; revision accepted Jun. 20, 2014

For correspondence or reprints contact: Thomas Reiner, Department of Radiology, Memorial Sloan-Kettering Cancer Center, 1275 York Ave., New York, NY 10065

E-mail: reinert@mskcc.org

Published online Jul. 24, 2014.

COPYRIGHT (C 2014 by the Society of Nuclear Medicine and Molecular Imaging, Inc.
$\mathbf{T}$ he value of nanoparticles and nanotechnology in clinical diagnostics (1), therapeutics (2,3), single-molecule (4) and singlecell (5) analysis, and systems-wide analysis (6) of clinical specimens has become virtually indispensable over the last few years. Nanomaterials are of interest for a wide array of different applications in biomedical settings because they can have extremely low detection thresholds (4) and can be used to amplify signals (7), enhance avidity (5), enhance binding (8), and translate biologic signals into measurable readouts for a variety of imaging technologies (9).

In particular, liposomal nanoparticles have been extensively exploited because of their modular structure, ease of modification, ability to encapsulate drugs and other payloads, and, importantly, efficient transport into tumors through the enhanced permeability and retention effect (10). The accumulation of liposomes via the enhanced permeability and retention effect is a noteworthy advantage in comparison to targeted therapies, since delivery of the liposomes is governed by a physical effect rather than the expression of a molecular marker. In addition, another potential mechanism for the delivery of nontargeted nanoparticles to tumors has recently been reported by which a particular subset of immune cells is partially responsible for the uptake, transport, and delivery of the nanomaterial to the cancer tissue (11). Consequently, taking advantage of these mechanisms (12-14) allows a single type of liposome to be used on fundamentally different types of cancer (3, 15). On the basis of their favorable biophysical properties, some liposomal drug formulations have found clinical application in cancer therapy (16). However, variations in tumor phenotype and the heterogeneity of enhanced permeability and retention often lead to variations in therapeutic response $(17,18)$. Therefore, the development of noninvasive screening methods could be of tremendous value in the assessment of amenability to nanotherapy for individual patients, which is still a major unmet clinical need (19, 20). Molecular imaging by PET is increasingly being exploited in drug development settings as a tool to prescreen candidates in early-stage trials by use of companion imaging agents (20). The potential health and economic impact of the application of this approach in the development of nanotherapeutics is easy to grasp. In the context of liposomal therapeutics, application requires a radiolabel 
that matches their long circulation times but also a high stability of the label over long periods. A labeling approach meeting these specifications would allow visualization of biodistribution of the liposomal nanoparticles and, therefore, assessment of the treatment outcome and safety. Radiolabeled liposomes with ${ }^{64} \mathrm{Cu}$ can be found in the literature $(21)$, but its half-life $(12.7 \mathrm{~h})$ may fall short of allowing an optimal imaging window in humans. ${ }^{89} \mathrm{Zr}$, on the other hand, has a half-life of $78.4 \mathrm{~h}$ and has been successfully used for the labeling and imaging of long-circulating probes such as antibodies in humans (22).

The use of dual-modality probes combining a PET label and a near-infrared (NIR) fluorophore brings together the best of both worlds, namely the high sensitivity and tissue penetration of PET and the cellular resolution of optical imaging. Successful examples using nanoparticles (23) and ${ }^{89} \mathrm{Zr}$-labeled antibodies (24) prove the value of this approach.

Here, we present a modular and highly efficient labeling strategy for the preparation of ${ }^{89} \mathrm{Zr}$-liposomes. Specifically, we constructed ${ }^{89} \mathrm{Zr}$-labeled liposomes via 2 different labeling strategies. One of them uses bioorthogonal labeling to link ${ }^{89} \mathrm{Zr}$ to the liposomes, whereas the other relies on the complexation of ${ }^{89} \mathrm{Zr}$ on the liposome surface with chelators. Additionally, we prepared a bimodal liposome for dual PET/optical imaging by incorporation of a NIR fluorescent label. We illustrate the differences that the 2 labeling strategies cause for the pharmacokinetic profile of the particles, and we prove that the incorporation of fluorescent dyes into the lipid bilayer can be achieved while maintaining the particles' performance.
Using xenograft and orthotopic models of breast cancer, we show that our single- and dual-modality liposomes have high target accumulation, are selective, and are well suited for PET and optical imaging techniques.

\section{MATERIALS AND METHODS}

A detailed description of synthetic procedures, preparation of liposomes and radiosynthesis, and all in vitro and in vivo experiments can be found in the supplemental data (supplemental materials are available at http:// jnm.snmjournals.org). All animal experiments were done in accordance with protocols approved by the Institutional Animal Care and Use Committee of Memorial Sloan-Kettering Cancer Center and followed National Institutes of Health guidelines for animal welfare.

\section{RESULTS}

\section{Synthesis of Functional Lipids}

The formulation of dibenzoazacyclooctyne (DBCO)-L and desferrioxamine (DFO)-L (Figs. 1A and 1B, respectively) required the synthesis of 2 derivatives of the phospholipid 1,2-distearoyl-sn-glycero3-phosphoethanolamine (DSPE, 1, Supplemental Fig. 1). Reaction of DSPE with the pegylated DBCO- $N$-hydroxysuccinimide ester $\mathbf{2}$ and the DFO-p-benzoisothiocyanate reagent $\mathbf{7}$ furnished compounds $\mathbf{3}$ and $\mathbf{8}$ in good yield (57\% and $85 \%$, respectively). Additionally, a clickable DFO-azide construct (6) was prepared using desferrioxamine mesylate $\mathbf{5}$ and the pegylated azide $N$-hydroxysuccinimide ester 4 (Supplemental Fig. 1).

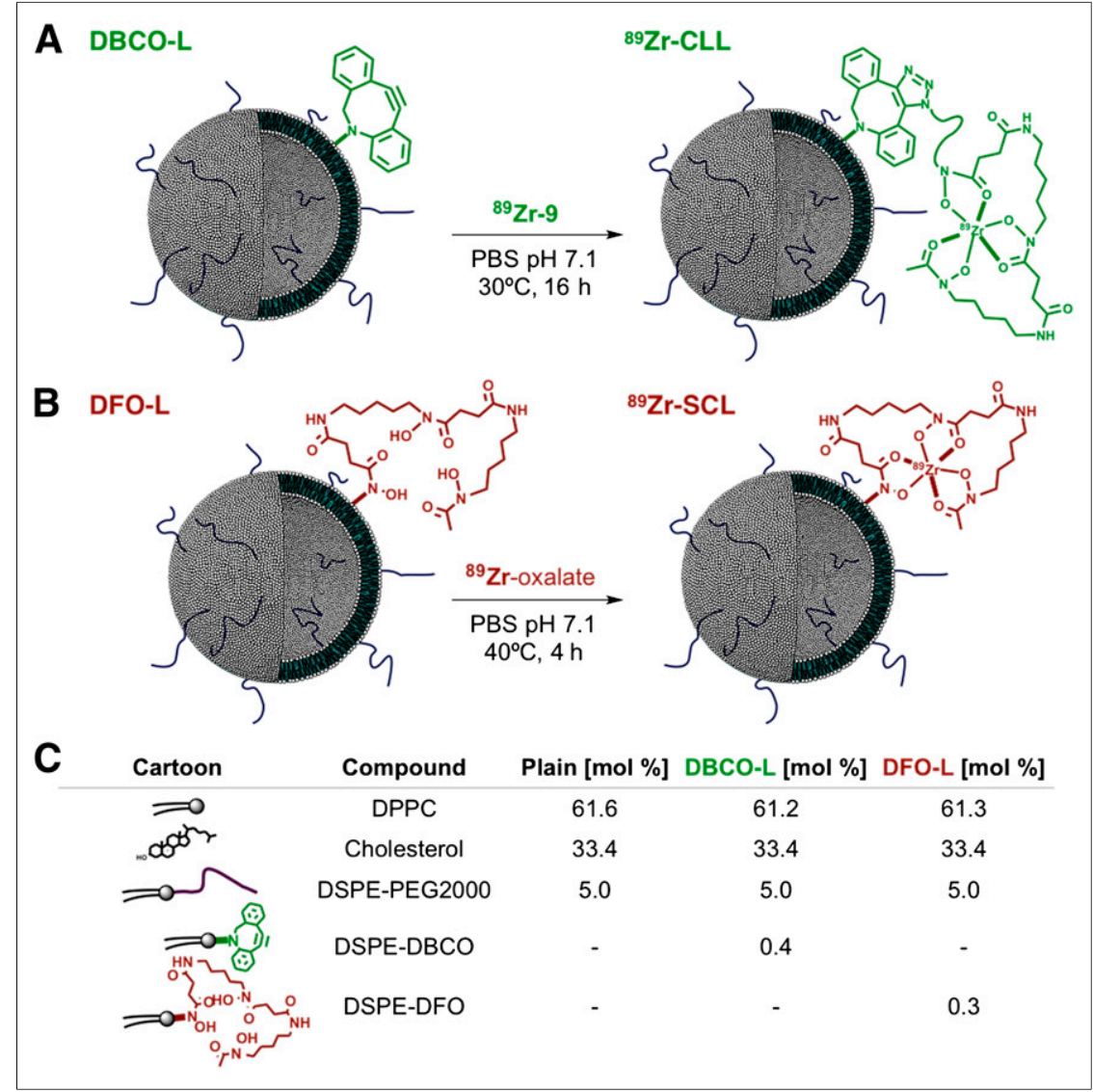

FIGURE 1. (A and B) Strategies for labeling of liposomes with ${ }^{89} \mathrm{Zr}$ by click labeling $(A)$ and surface chelation (B). (C) Lipid composition of liposomes used in present work. PBS = phosphatebuffered saline.

\section{Formulation of Liposomal Nanoparticles}

Nonfunctionalized liposomes composed of 1,2-dipalmitoyl-sn-glycero-3-phosphocholine (DPPC), cholesterol, and pegylated DSPE (DSPE-PEG2000) in a 1.85:1:0.15 molar ratio were prepared by adding the individual lipids to a reaction vessel in a solution of chloroform, removing the organic solvent, and adding phosphate-buffered saline, followed by sonication and centrifugation. This procedure yielded liposomal nanoparticles with a mean diameter of $100 \mathrm{~nm}$ and a low polydispersity (mean effective diameter [MED] \pm SD: $103.4 \pm 5.1 \mathrm{~nm}$, polydispersity index [PDI]: $0.11 \pm 0.01, n=4$ ), with a slightly negatively charged surface ( $Z$ potential: $-21.7 \pm 5.4 \mathrm{mV}, n=3$ ). The functionalized counterparts of the nonlabeled liposomes, DBCO-L and DFO-L, were prepared identically but had the lipids $\mathbf{3}$ and $\mathbf{8}$ added to their respective initial lipid mixtures $(0.4 \%$ for DBCO-L and $0.3 \%$ for DFO-L), at the expense of DPPC (Fig. 1C). The presence of the new lipids was well tolerated, and the size and polydispersity of the resulting liposomes were comparable to those of the plain liposomes $\left(\mathrm{MED}_{\mathrm{DFO}-\mathrm{L}}\right.$ : $102.7 \pm 4.6 \mathrm{~nm}, \mathrm{PDI}_{\mathrm{DFO}-\mathrm{L}}: 0.14 \pm 0.02 \mathrm{~nm}$, $n=6$; MED $_{\text {DвCO-L }}: 102.3 \pm 1.4 \mathrm{~nm}$, $\mathrm{PDI}_{\mathrm{DBCO}-\mathrm{L}}: 0.12 \pm 0.01 \mathrm{~nm}, n=3$, Supplemental Fig. 2A). Both preparations were stable for weeks at $4^{\circ} \mathrm{C}$, with no measurable 


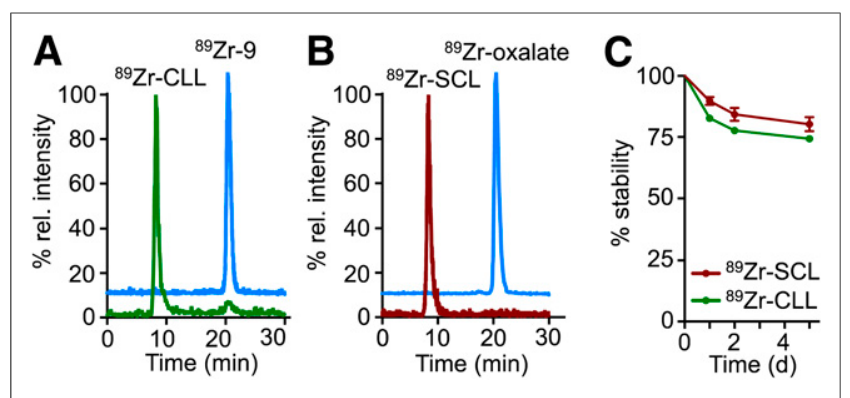

FIGURE 2. (A and B) Size-exclusion chromatograms showing radioactive traces of ${ }^{89} \mathrm{Zr}$-CLL and ${ }^{89} \mathrm{Zr}-9$ (A) and ${ }^{89} \mathrm{Zr}$-SCL and ${ }^{89} \mathrm{Zr}$-oxalate (B). (C) In vitro serum stability of ${ }^{89} \mathrm{Zr}-\mathrm{CLL}$ and ${ }^{89} \mathrm{Zr}-\mathrm{SCL}$.

aggregation or loss of reactivity. The surface charge was not substantially affected either, although a slight variation toward neutrality was observed for DBCO-L ( $Z$ potential: $-22.6 \pm 3.9 \mathrm{mV}$, $n=4$, for DFO-L; $-15.9 \pm 3.1 \mathrm{mV}, n=2$, for DBCO-L).

\section{Radiolabeling and Stability of Liposomes}

The preparation of click-labeled liposomes ( ${ }^{89} \mathrm{Zr}-\mathrm{CLL}$, Fig. 1A) required the labeling of the bioorthogonally reactive DFO derivative 6 with ${ }^{89} \mathrm{Zr}$, which was obtained in $44 \% \pm 15 \%$ yield after isolation by high-performance liquid chromatography (HPLC) (Supplemental Fig. 3). The isolated labeled fragment, ${ }^{89} \mathrm{Zr}-9$, was then reacted with DBCO-L overnight at $30^{\circ} \mathrm{C}$ (Fig. 1A). Purification of ${ }^{89} \mathrm{Zr}$-CLL by spin filtration afforded the radiolabeled material in $14 \% \pm 4 \%$ radiochemical yield and more than $95 \%$ purity (Fig. 2A).
The labeling process did not affect the distribution (size and PDI) of the sample, as compared with DBCO-L (MED ${ }^{8 \mathrm{r}-\mathrm{CLL}}$ : $106.4 \pm 7.3$, $\mathrm{PDI}^{89}{ }_{\text {Zr-CLL }}: 0.12 \pm 0.02, n=3$, Supplemental Fig. $2 \mathrm{~A}$ ). In a control experiment, nonfunctionalized liposomes did not take up or adsorb any radioactivity when incubated under the same conditions with ${ }^{89} \mathrm{Zr}-9$. When ${ }^{89} \mathrm{Zr}$-CLL was disassembled after the addition of ethanol, we were able to detect the radiolabeled clicked lipid by radio-HPLC, confirming successful bioorthogonal ligation.

The radiolabeling of liposomes by surface chelation was achieved by incubation of DFO-L (Fig. 1B) with an ${ }^{89} \mathrm{Zr}$-oxalate solution in phosphate-buffered saline at $\mathrm{pH} 7.1-7.4$ for $4 \mathrm{~h}$ at $40^{\circ} \mathrm{C}$ and purified by spin filtration. The radiochemical yield was $80 \% \pm 10 \%$ $(n=7)$ and the resulting liposomes, ${ }^{89} \mathrm{Zr}$-surface-chelation (labeled) liposomes (SCL), were radiochemically pure (Fig. 2B). No statistically significant increase in the size of the liposomes was observed after labeling, compared with precursor DFO-L $\left(\mathrm{MED}^{89}{ }_{\mathrm{Zr}-\mathrm{SCL}}: 108.5 \pm 4.6, \mathrm{PDI}^{89} \mathrm{Zr-SCL}: 0.15 \pm 0.02, n=6\right.$, Supplemental Fig. 2A). A control experiment was performed to assess the binding of ${ }^{89} \mathrm{Zr}$ to the surface of plain, nonfunctionalized liposomes. Incubation of a sample of these liposomes with ${ }^{89} \mathrm{Zr}$ oxalate at $40^{\circ} \mathrm{C}$ over a period of $16 \mathrm{~h}$ afforded less than $0.5 \%$ of activity bound to liposomes. The radiolabeled lipid was also detected by radio-HPLC analysis of a sample of disassembled liposomes.

The stability of both labeled liposome preparations was assessed in serum (fetal bovine serum) by incubation of samples at $37^{\circ} \mathrm{C}$ for a period of $5 \mathrm{~d}$ (Fig. 2C). Release of activity (10\% for ${ }^{89} \mathrm{Zr}$-SCL and $17 \%$ for ${ }^{89} \mathrm{Zr}$-CLL, after $24 \mathrm{~h}$ ) was observed for both labeling strategies. At later time points, a fraction of plasma proteins with an estimated molecular weight of $40 \mathrm{kDa}$ took up some of the liberated activity $(<3 \%$ and $<6 \%$ after $48 \mathrm{~h}$ and $120 \mathrm{~h}$, respectively). However, it remains challenging to establish whether this effect is based on the chelation of free ${ }^{89} \mathrm{Zr}$ by the plasma protein fraction or on lipid exchange between the liposomes and plasma proteins. Overall, however, these data indicate a high stability of the chelator on the lipid surface of both ${ }^{89} \mathrm{Zr}$-SCL and ${ }^{89} \mathrm{Zr}-\mathrm{CLL}$ (Fig. 2C).

\section{Pharmacokinetics and In Vivo Imaging with ${ }^{89} \mathrm{Zr}$-Labeled Liposomes}

In vivo evaluation of both labeled liposomes started with the measurement of their blood half-life in healthy female $\mathrm{NCr}$ nude mice (Fig. 3A). The weighted half-life of ${ }^{89} \mathrm{Zr}$-SCL proved to be markedly longer than that of ${ }^{89} \mathrm{Zr}-\mathrm{CLL}\left(\mathrm{t}_{1 / 2}=7.20 \mathrm{~h}\right.$ and $1.25 \mathrm{~h}$, respectively). Figure $3 \mathrm{~B}$ shows a comparative ${ }^{89} \mathrm{Zr}$ activity biodistribution in selected tissues after intravenous administration of the liposomes in mice bearing 4T1 breast cancer xenografts, which were chosen as a representative model of solid tumors (Supplemental Tables 1 and 2).

$\mathrm{PET} / \mathrm{CT}$ imaging with ${ }^{89} \mathrm{Zr}-\mathrm{CLL}$ at $2 \mathrm{~h}$ after injection showed predominantly liver and spleen uptake. There was no substantial difference at $24 \mathrm{~h}$ after injection (Supplemental Fig. 4) and subsequent time points. In contrast, ${ }^{89} \mathrm{Zr}$-SCL PET images at first
FIGURE 3. (A) Pharmacokinetics of ${ }^{89} \mathrm{Zr}$-SCL and ${ }^{89} \mathrm{Zr}$-CLL $(n=3)$. (B) Radioactivity distribution in selected tissues of ${ }^{89} \mathrm{Zr}$-SCL and ${ }^{89} \mathrm{Zr}$-CLL $(n \geq 3)$. (C) PET/CT imaging of ${ }^{89} \mathrm{Zr}$-SCL: CT only (left), PET/CT fusion (middle), and 3-dimensional rendering of PET/CT fusion (right) at $24 \mathrm{~h}$ after injection.
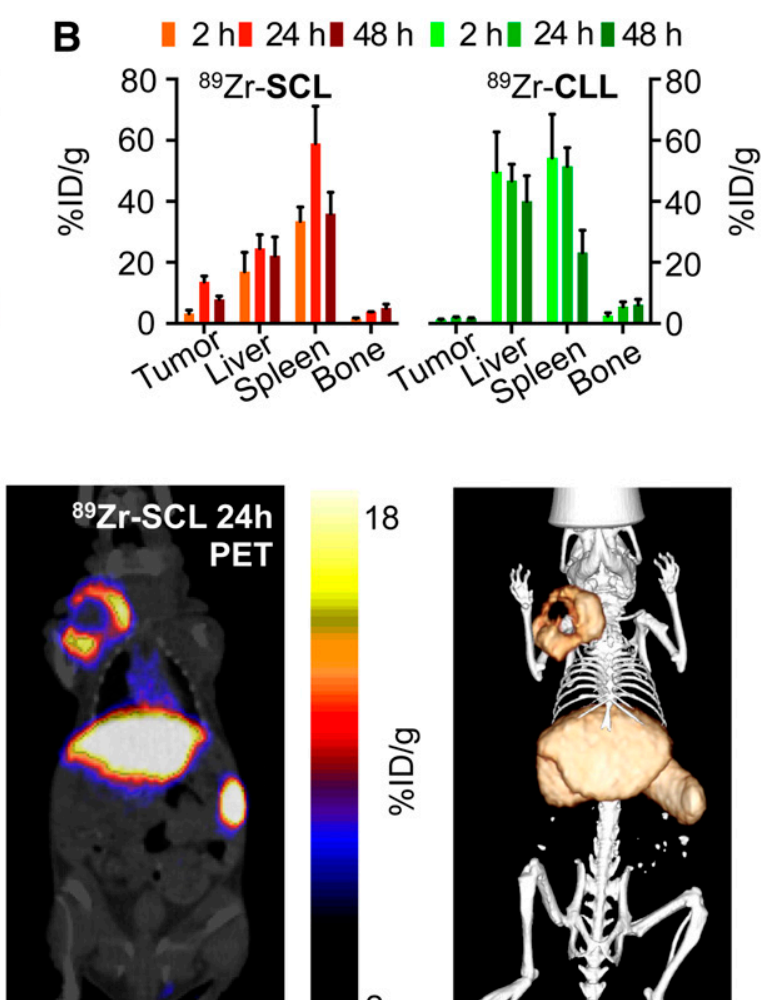

18

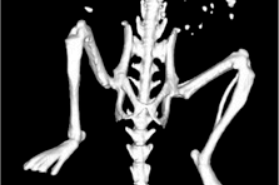


showed high blood-pool activity $(24.3 \pm 4.2$ percentage injected dose per gram of tissue [\% $\% \mathrm{D} / \mathrm{g}$ ] in heart, $n=2$ ) and strong signals from liver and spleen. At $24 \mathrm{~h}$, the blood-pool signal was moderate (6.2 $\pm 0.4 \% \mathrm{ID} / \mathrm{g}$ in heart, $n=4)$, but tumor accumulation was considerably higher. In accordance with the biodistribution results, spleen and liver tissues showed the highest uptake at all time points. Quantitative data obtained from PET scans agreed well with the biodistribution results. Figure $3 \mathrm{C}$ shows that the overall tumor uptake at $24 \mathrm{~h}$ was high, measuring $14.1 \pm 1.6 \% \mathrm{ID} / \mathrm{g}(n=4)$. Later time points showed blood activity clearance but persistent PET signal in tumor $(12.7 \pm 1.0$ and $10.2 \pm 0.5 \% \mathrm{ID} / \mathrm{g}, n=2$, at $48 \mathrm{~h}$ and $120 \mathrm{~h}$, respectively).

\section{Histology}

Ex vivo analysis by autoradiography and histologic staining of tumor sections (excised at $24 \mathrm{~h}$ after administration of ${ }^{89} \mathrm{Zr}-\mathrm{SCL}$ ) was performed to elucidate the regional distribution of the radiotracer (Supplemental Fig. 5). Hematoxylin and eosin staining (Supplemental Fig. 5A) revealed an inner region characterized by a reduced number of nuclei and viable cells, consistent with a developing necrotic core (Supplemental Fig. 5D), as opposed to the external areas, which showed a higher cell density and normal appearance (Supplemental Fig. 5E). The inner region did not stain for IBA-1 (ionized calcium binding adaptor molecule 1, Supplemental Fig. $5 \mathrm{~B}$ ), which is specifically expressed in macrophages and microglia, and had a low accumulation of ${ }^{89} \mathrm{Zr}$-SCL (Supplemental Fig. 5C).

\section{Bimodal Imaging with Cy5/89 Zr-Labeled Liposomes}

A ready-to-label liposome incorporating the NIR dye Cy5 analog 1,1-diododecyl-3,3,3,3-tetramethyl-indodicarbocyanine-5,5disulfonic acid (DiIC12 (5)-DS), namely DiIC@DFO-L, was labeled with ${ }^{89} \mathrm{Zr}$ following the same procedure used for ${ }^{89} \mathrm{Zr}-\mathrm{SCL}$, yielding the bimodal liposome DiIC@ ${ }^{89} \mathrm{Zr}-\mathrm{SCL}$ (Fig. 4A) in $88 \%$ radiochemical yield and more than $99 \%$ radiochemical purity (Supplemental Fig. 2B). Subsequent PET imaging showed that the fluorescent DiIC@ ${ }^{89} \mathrm{Zr}$-SCL was also long-circulating and had essentially the same performance as ${ }^{89} \mathrm{Zr}$-SCL (Supplemental Fig. 6A). Histologic analysis of tumor sections revealed a high

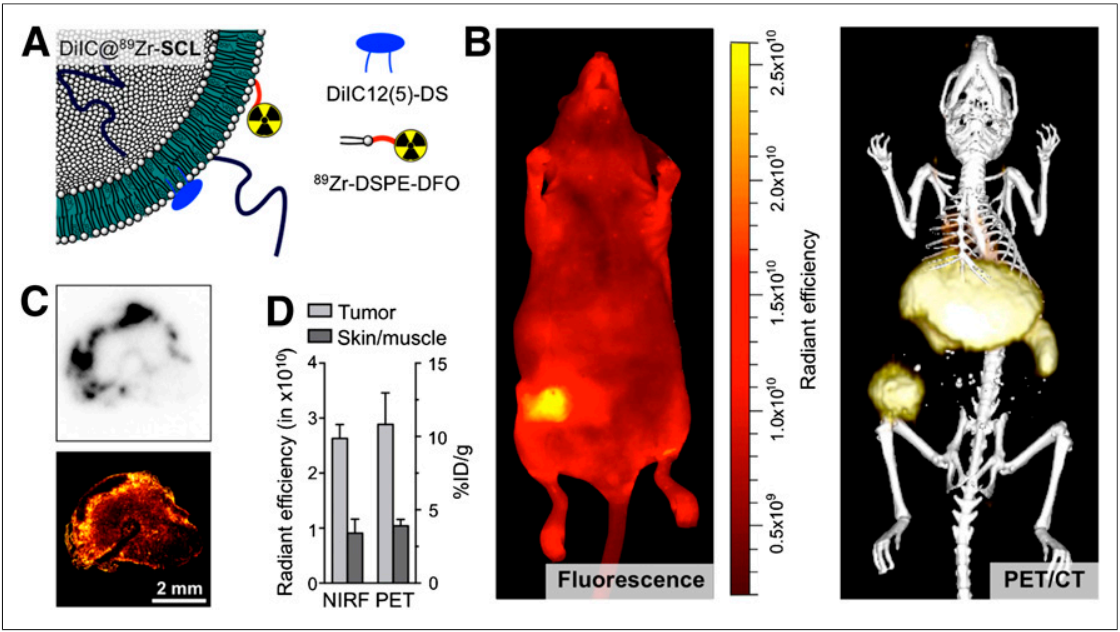

FIGURE 4. (A) Schematic of dual-labeled liposome DilC@89Zr-SCL. (B) Whole-body NIR fluorescence imaging (excitation wavelength $=650 \mathrm{~nm} / \mathrm{emission}$ wavelength $=670 \mathrm{~nm}$ ) (left) and 3dimensional rendering of PET/CT fusion image (right) of same animal at $24 \mathrm{~h}$ after administration of DilC@ ${ }^{89} \mathrm{Zr}$-SCL. (C) Tumor sections showing autoradiography (top) and confocal microscopy at $670 \mathrm{~nm}$ (bottom). (D) Comparison of NIR fluorescence (NIRF) and PET quantification measurements in tumor and skin areas (skin-to-muscle ratio for PET; $n=3$ ). level of colocalization of radioactivity and fluorescence, as shown in Figure 4C. Both signals were localized to the periphery of the tissue, indicating the progress of an incipient necrotic core. Furthermore, same-animal analysis $(n=3)$ of PET/CT and epifluorescence NIR imaging at $24 \mathrm{~h}$ after injection (Fig. 4B) yielded an excellent correlation between the 2 imaging modalities (Fig. 4D) on the whole-body level. The tumor-to-skin (fluorescence)/skinto-muscle (PET) ratios were measured to be 2.8 and 2.9, respectively, using PET/CT and NIR fluorescent data and compared well with the values obtained from biodistribution experiments (tumorto-skin, 2.3).

\section{DISCUSSION}

The goal of this study was to develop a modular and efficient radiolabeling technology for long-circulating liposomal nanoparticles. Over the last few years, a wealth of bioorthogonal labeling techniques has been developed and successfully used to prepare, radiolabel, and functionalize diverse agents (25-27). In our search for an efficient bioorthogonal approach to label liposomes, we were interested mostly in those that could provide efficient conjugation without the need for catalysts. And although the strain-promoted, copper-free alkyne-azide reaction rates are generally slower than those of other bioorthogonal reactions (28), the higher stability of the reactive bioorthogonal building blocks makes this approach superior for applications that demand challenging or harsh synthetic conditions (29). Therefore, we decided to prepare DBCO-containing liposomes (DBCO-L, Fig. 1A) for click labeling and compared this approach with a direct labeling method (Fig. 1B), the surface chelation of ${ }^{89} \mathrm{Zr}$ on DFO-bearing liposomes (DFO-L). Figure $1 \mathrm{C}$ lists the necessary components for the formulation of the 2 different types of liposomes.

All liposomes were larger than the renal clearance threshold (30). The overall negative charge of the particles helps to stabilize them in vivo, reducing the tendency to aggregate and avoiding electrostatic interactions with the luminal wall of blood vessels (10). Moreover, the presence of polyethylene glycol chains on the surface of the particles efficiently helps to prevent opsonization and their subsequent removal from circulation (31).

Compared with the bioorthogonal labeling, direct surface chelation proved to be quicker and more efficient, but neither strategy had a significant effect on the size distribution of the samples when compared with their precursor liposomes. Although both probes had a similar size, the clearance rates of radioactivity from blood were significantly different. After 24 h, $7.1 \% \mathrm{ID} / \mathrm{g}$ remained in the blood pool for ${ }^{89} \mathrm{Zr}-\mathrm{SCL}$, whereas the activity for ${ }^{89} \mathrm{Zr}-\mathrm{CLL}$ had dropped to $1.5 \% \mathrm{ID} / \mathrm{g}$. Circulation time is a critical factor for tumor accumulation, especially for probes reliant on passive targeting mechanisms. Higher accumulation is to be expected for those species showing the longest half-lives, as more particles will extravasate into the interstitial space with higher passage numbers (10). The fast blood clearance observed for ${ }^{89} \mathrm{Zr}$-CLL can be explained by the rapid accumulation of radioactivity in liver and spleen, which 
are the organs that most efficiently remove particles from circulation $(32,33)$. In contrast, ${ }^{89} \mathrm{Zr}$-SCL seemed to evade the mononuclear phagocyte system (34) longer and therefore had substantially lower liver and spleen uptake at $2 \mathrm{~h}$ after injection. This difference could be due to a higher tendency of ${ }^{89} \mathrm{Zr}$-CLL to aggregate into larger particles and their subsequent removal by the mononuclear phagocyte system and illustrates how changes in the surface chemistry of nanoparticles can have far-reaching consequences on their stability and pharmacokinetic profile. As a result, tumor accumulation was dramatically higher for ${ }^{89} \mathrm{Zr}$-SCL than for their clicklabeled counterpart ${ }^{89} \mathrm{Zr}$-CLL at all time points, peaking at $24 \mathrm{~h}$ after injection $(13.7 \pm 1.8 \% \mathrm{ID} / \mathrm{g}$ and $2.0 \pm 0.2 \% \mathrm{ID} / \mathrm{g}$, respectively). Tumor-to-blood uptake ratios for ${ }^{89} \mathrm{Zr}$-SCL were $0.09,2.0$, and 4.2 at 2,24 , and $48 \mathrm{~h}$, respectively. Interestingly, no correlation was found between size of tumor and uptake $(\% \mathrm{ID} / \mathrm{g})$ for either probe. For both liposomes, bone uptake was moderate $(<6 \% \mathrm{ID} / \mathrm{g}$ at $48 \mathrm{~h}$ ), which indicates that the ${ }^{89} \mathrm{Zr}$-DFO metal complex in the liposome surface is rather protected from transchelation and release of the radiotracer. These data also indirectly support the low unspecific adsorption of ${ }^{89} \mathrm{Zr}$ on the surface of ${ }^{89} \mathrm{Zr}$-SCL, as liposomes labeled in the absence of DFO or other chelators have poorer in vitro and in vivo stabilities and, consequently, higher bone uptake (35). The data presented here compare well with reported bone uptake values for other long-circulating ${ }^{89} \mathrm{Zr}$-labeled probes $(36,37)$.

PET/CT imaging mirrored quite precisely the results of the biodistribution studies for both liposomes as shown in mice bearing either xenografted or orthotopically implanted 4T1 breast tumors. Although low tumor uptake could be observed for ${ }^{89} \mathrm{Zr}$-CLL, an intense and persistent signal was found in all tumors imaged with ${ }^{89} \mathrm{Zr}$-SCL at $24 \mathrm{~h}$ (Fig. 3C) and subsequent time points. There was no statistically significant difference observed between uptake in xenografts and orthotopically implanted tumors at $24 \mathrm{~h}$ after administration of ${ }^{89} \mathrm{Zr}-\mathrm{SCL}(13.1 \pm 1.8 \% \mathrm{ID} / \mathrm{g}, n=4$, and $12.2 \pm$ $3.4 \% \mathrm{ID} / \mathrm{g}, n=3$, respectively). PET-quantified bone uptake was also similar to the biodistribution data for both formulations and was found to be lower than $5 \% \mathrm{ID} / \mathrm{g}$ at all time points, even after $120 \mathrm{~h}$. The radioisotope ${ }^{89} \mathrm{Zr}$ is a bone seeker that eventually will accumulate in the mineral bone if released from its chelator DFO. A second pathway that might result in bone accumulation of nanoparticle-bound radiotracers is their uptake in macrophages. The presence of tissue macrophages in the bone marrow as part of the mononuclear phagocyte system makes the bone marrow a potential undesired destination for radiolabeled nanoparticles (38).

The distribution of ${ }^{89} \mathrm{Zr}$-SCL in the tumors was not homogeneous (Fig. 3C). In tumors grown for over $10 \mathrm{~d}, 2$ regions were clearly distinguishable: a peripheral shell with high accumulation (as high as $20 \% \mathrm{ID} / \mathrm{g}$ ) and a central core showing low tracer uptake. Histologic analysis confirmed these findings (Supplemental Fig. 5), and there is evidence for localization of ${ }^{89} \mathrm{Zr}-\mathrm{SCL}$ in macrophage-rich areas, as reported for other nanoparticulate systems in varied disease models (38).

Encouraged by the results obtained with ${ }^{89} \mathrm{Zr}-\mathrm{SCL}$, we aimed to generate a bimodal PET/optical imaging agent by adding an NIR fluorescent dye (DiIC) to the lipid formulation. Although tumor uptake for DiIC@ ${ }^{89} \mathrm{Zr}$-SCL as measured by PET was lower (10.8 \pm $2.1 \% \mathrm{ID} / \mathrm{g}, n=3)$ than that for ${ }^{89} \mathrm{Zr}-\mathrm{SCL}(14.1 \pm 1.6 \% \mathrm{ID} / \mathrm{g}, n=$ $4)$, this difference was not statistically significant $(P=0.10)$. The high level of colocalization of both signals (Fig. 4C), as well as the quantitative analyses by PET and NIR fluorescence imaging (Fig. 4D), suggest a good stability of these liposomal nanoparticles.

\section{CONCLUSION}

This study presents a highly modular and efficient route for the synthesis of long-circulating ${ }^{89} \mathrm{Zr}$-labeled liposomes. The resulting nanomaterial, ${ }^{89} \mathrm{Zr}-\mathrm{SCL}$, has demonstrated an outstanding ability to target xenografts and orthotopic mouse models of breast cancer in vivo. Moreover, our nanoplatform was able to accommodate a NIR dye to yield a PET/optical bimodal probe without detriment to its ability to target malignancy. We believe that the impact of the application of the technology described herein is manifold, as it can readily be adapted for other lipidic nanomaterials, including micelles, nanoemulsions, and lipoprotein nanoparticles. In a translational scenario, its obvious potential use as a companion imaging agent makes it an ideal candidate for the evaluation of the safety and efficacy of nanotherapeutics. In addition, with the advent of modern intravital imaging and the promises these techniques hold when translated to the clinic (39), anatomic imaging protocols are quickly moving toward the combination of intraoperative and whole-body imaging techniques, enabling radiologists to detect both the presence of lesions on a whole-body level and the exact extent of disease at the operating table. In this regard, given its proven versatility, our nanoplatform seems to meet the requirements to fulfill this task. In a wider disease context, we believe that studies to assess its potential application in other maladies and ultimately in a clinical setting are warranted.

\section{DISCLOSURE}

The costs of publication of this article were defrayed in part by the payment of page charges. Therefore, and solely to indicate this fact, this article is hereby marked "advertisement" in accordance with 18 USC section 1734. This work was supported by the NIH (K25 EB016673 and R01 CA155432), the Brain Tumor Center of Memorial Sloan-Kettering Cancer, the Nanotechnology Center for Molecular Imaging and Nanotechnology, and the CNIC CardioImage program. No other potential conflict of interest relevant to this article was reported.

\section{ACKNOWLEDGMENTS}

We thank the Small Animal Imaging Core, the Radiochemistry and Molecular Imaging Probes Core, and the Molecular Cytology Core at Memorial Sloan-Kettering Cancer Center for support. We also thank Sean Carlin for his help with histological analysis and Sarah M. Cheal for helpful discussions on PET imaging analysis.

\section{REFERENCES}

1. Cheng MM, Cuda G, Bunimovich YL, et al. Nanotechnologies for biomolecular detection and medical diagnostics. Curr Opin Chem Biol. 2006;10:11-19.

2. Schroeder A, Heller DA, Winslow MM, et al. Treating metastatic cancer with nanotechnology. Nat Rev Cancer. 2012;12:39-50.

3. Allen TM, Cullis PR. Liposomal drug delivery systems: from concept to clinical applications. Adv Drug Deliv Rev. 2013;65:36-48.

4. Jin H, Heller DA, Kalbacova M, et al. Detection of single-molecule $\mathrm{H}_{2} \mathrm{O}_{2}$ signalling from epidermal growth factor receptor using fluorescent single-walled carbon nanotubes. Nat Nanotechnol. 2010;5:302-309.

5. Tassa C, Duffner JL, Lewis TA, et al. Binding affinity and kinetic analysis of targeted small molecule-modified nanoparticles. Bioconjug Chem. 2010;21:14-19.

6. Fan R, Vermesh O, Srivastava A, et al. Integrated barcode chips for rapid, multiplexed analysis of proteins in microliter quantities of blood. Nat Biotechnol. 2008;26:1373-1378.

7. Haun JB, Devaraj NK, Hilderbrand SA, Lee H, Weissleder R. Bioorthogonal chemistry amplifies nanoparticle binding and enhances the sensitivity of cell detection. Nat Nanotechnol. 2010;5:660-665.

8. Shaw SY, Westly EC, Pittet MJ, Subramanian A, Schreiber SL, Weissleder R. Perturbational profiling of nanomaterial biologic activity. Proc Natl Acad Sci USA. 2008;105:7387-7392. 
9. Cai W, Chen X. Nanoplatforms for targeted molecular imaging in living subjects. Small. 2007;3:1840-1854.

10. Maeda H, Nakamura H, Fang J. The EPR effect for macromolecular drug delivery to solid tumors: improvement of tumor uptake, lowering of systemic toxicity, and distinct tumor imaging in vivo. Adv Drug Deliv Rev. 2013;65: 71-79.

11. Smith BR, Ghosn EE, Rallapalli H, et al. Selective uptake of single-walled carbon nanotubes by circulating monocytes for enhanced tumour delivery. Nat Nanotechnol. 2014;9:481-487.

12. Matsumura Y, Maeda H. A new concept for macromolecular therapeutics in cancer chemotherapy: mechanism of tumoritropic accumulation of proteins and the antitumor agent smancs. Cancer Res. 1986;46:6387-6392.

13. Schroeder A, Kost J, Barenholz Y. Ultrasound, liposomes, and drug delivery: principles for using ultrasound to control the release of drugs from liposomes. Chem Phys Lipids. 2009;162:1-16.

14. Chauhan VP, Stylianopoulos T, Martin JD, et al. Normalization of tumour blood vessels improves the delivery of nanomedicines in a size-dependent manner. Nat Nanotechnol. 2012;7:383-388.

15. Luk BT, Fang RH, Zhang L. Lipid- and polymer-based nanostructures for cancer theranostics. Theranostics. 2012;2:1117-1126.

16. Torchilin VP. Recent advances with liposomes as pharmaceutical carriers. Nat Rev Drug Discov. 2005;4:145-160.

17. Mullard A. Molecular imaging as a de-risking tool: coming into focus? Nat Rev Drug Discov. 2013;12:251-252.

18. Hargreaves RJ. The role of molecular imaging in drug discovery and development. Clin Pharmacol Ther. 2008;83:349-353.

19. Prabhakar U, Maeda H, Jain RK, et al. Challenges and key considerations of the enhanced permeability and retention effect for nanomedicine drug delivery in oncology. Cancer Res. 2013;73:2412-2417.

20. Sanhai WR, Sakamoto JH, Canady R, Ferrari M. Seven challenges for nanomedicine. Nat Nanotechnol. 2008;3:242-244.

21. Petersen AL, Binderup T, Rasmussen P, et al. ${ }^{64} \mathrm{Cu}$ loaded liposomes as positron emission tomography imaging agents. Biomaterials. 2011;32:2334-2341.

22. Gaykema SB, Brouwers AH, Lub-de Hooge MN, et al. ${ }^{89} \mathrm{Zr}$-bevacizumab PET imaging in primary breast cancer. J Nucl Med. 2013;54:1014-1018.

23. Nahrendorf M, Keliher E, Marinelli B, et al. Hybrid PET-optical imaging using targeted probes. Proc Natl Acad Sci USA. 2010;107:7910-7915.

24. Cohen R, Vugts DJ, Stigter-van Walsum M, Visser GW, van Dongen GA. Inert coupling of IRDye800CW and zirconium-89 to monoclonal antibodies for single- or dual-mode fluorescence and PET imaging. Nat Protoc. 2013;8: 1010-1018.

25. Algar WR, Prasuhn DE, Stewart MH, et al. The controlled display of biomolecules on nanoparticles: a challenge suited to bioorthogonal chemistry. Bioconjug Chem. 2011;22:825-858

26. Reiner T, Zeglis BM. The inverse electron demand Diels-Alder click reaction in radiochemistry. J Labelled Comp Radiopharm. 2014;57:285-290.

27. Zeng D, Zeglis BM, Lewis JS, Anderson CJ. The growing impact of bioorthogonal click chemistry on the development of radiopharmaceuticals. J Nucl Med. 2013;54:829-832.

28. Wong CH, Zimmerman SC. Orthogonality in organic, polymer, and supramolecular chemistry: from Merrifield to click chemistry. Chem Commun (Camb). 2013;49:1679-1695.

29. Liang Y, Mackey JL, Lopez SA, Liu F, Houk KN. Control and design of mutual orthogonality in bioorthogonal cycloadditions. J Am Chem Soc. 2012;134: 17904-17907.

30. Choi HS, Liu W, Misra P, et al. Renal clearance of quantum dots. Nat Biotechnol. 2007;25:1165-1170.

31. Jokerst JV, Lobovkina T, Zare RN, Gambhir SS. Nanoparticle PEGylation for imaging and therapy. Nanomedicine (Lond). 2011;6:715-728.

32. Riviere JE. Pharmacokinetics of nanomaterials: an overview of carbon nanotubes, fullerenes and quantum dots. Wiley Interdiscip Rev Nanomed Nanobiotechnol. 2009;1:26-34.

33. Almeida JP, Chen AL, Foster A, Drezek R. In vivo biodistribution of nanoparticles. Nanomedicine (Lond). 2011;6:815-835.

34. Longmire M, Choyke PL, Kobayashi H. Clearance properties of nano-sized particles and molecules as imaging agents: considerations and caveats. Nanomedicine (Lond). 2008;3:703-717.

35. Abou DS, Thorek DL, Ramos NN, et al. ${ }^{89} \mathrm{Zr}$-Labeled paramagnetic octreotideliposomes for PET-MR imaging of cancer. Pharm Res. 2013;30:878-888.

36. Zeglis BM, Mohindra P, Weissmann GI, et al. Modular strategy for the construction of radiometalated antibodies for positron emission tomography based on inverse electron demand Diels-Alder click chemistry. Bioconjug Chem. 2011;22:2048-2059.

37. Chang AJ, Desilva R, Jain S, Lears K, Rogers B, Lapi S. ${ }^{89} \mathrm{Zr}$-radiolabeled trastuzumab imaging in orthotopic and metastatic breast tumors. Pharmaceuticals (Basel). 2012;5:79-93.

38. Weissleder R, Nahrendorf M, Pittet MJ. Imaging macrophages with nanoparticles. Nat Mater. 2014;13:125-138.

39. Pittet MJ, Weissleder R. Intravital imaging. Cell. 2011;147:983-991. 